\title{
A new design approach for customised medical devices realized by additive manufacturing
}

\author{
V. Ricotta ${ }^{1} \cdot$ R. I. Campbell ${ }^{2} \cdot$ T. Ingrassia $^{1}\left(\mathbb{D} \cdot\right.$ V. Nigrelli $^{1}$
}

Received: 14 May 2020 / Accepted: 28 August 2020 / Published online: 7 September 2020

(c) The Author(s) 2020

\begin{abstract}
The aim of this work is the design of a new customised elbow orthosis completely realized by Additive Manufacturing and the development of generative algorithms for parametric modelling and creation of 3D patterns to be adapted to the CAD model. This work describes a method to perfect the design of a custom elbow orthosis. A reverse engineering approach has been used to digitalize the patient's arm and the subsequent CAD modelling of the structure of the custom elbow orthosis has been performed. In particular, two algorithms have been implemented for the creation of 3D patterns and Voronoi tessellations. Subsequently, FEM analyses have been carried out to validate the design. Finally, a prototype of the elbow orthosis with Voronoi tessellation has been realized by means of the SLS technology. The results obtained have demonstrated that the implemented algorithm solved the problems found during CAD modelling with conventional software. Furthermore, the results of FEM analyses have validated the design choices. All this allowed realizing the prototype by AM technologies without problems. Moreover, the new proposed modelling approaches allows creating, in an interactive way, patterns on complex surfaces. The results of this research activity present innovative elements of originality in the CAD modelling sector, which can contribute to solving problems related to the modelling for Additive Manufacturing. Furthermore, another innovative characteristic of the device is the use of torsion springs that simulate the action of physiotherapists during exercises for patient rehabilitation.
\end{abstract}

Keywords Reverse engineering $\cdot$ Additive manufacturing $\cdot$ Generative design $\cdot$ Computer aided design $\cdot$ Elbow orthosis

\section{Introduction}

Orthoses are medical devices that help people with disabilities. Orthoses support, align, prevent or correct deformities and improve the joint function with the purpose of improving the users' quality of life. For patients with disabilities that contribute to functional limitations, orthoses are used to apply forces on the body for biomechanical needs [1-3]. Orthoses can be divided into two main categories: the nonarticular and the articular. The articulated orthoses permit an adjustment during their use while the non-articulated are the ones which have only one possible configuration and, consequently, do not allow a regulation during their use [4]. Moreover, orthoses can be customised, adapting better to

T. Ingrassia

tommaso.ingrassia@unipa.it

1 Dipartimento di Ingegneria, Università degli Studi di Palermo, Viale delle Scienze, 90128 Palermo, Italy

2 Loughborough Design School, Loughborough University, Loughborough LE11 3TU, UK the patient's body than the prefabricated devices and ensuring better performances [5-7]. The fitness of orthoses is the most important factor for patients' satisfaction. Traditionally, custom orthoses are manufactured using a labour intensive plaster molding technique [1].

Additive manufacturing (AM) is an ideal technology for mass customization and provides the opportunity to eliminate much of this labour. AM is a suitable technology to make customised devices, especially for its ability to realize complex shapes in a short time and with lower costs than manual fabrication [8-10] and a good surface finish depending on the process parameters [11]. The applications of AM have increased extensively in the area of orthopaedics. It gives a flexible solution in orthopaedics area, where customised implants can be formed as per the required shape and size and can help substitution with customised products.

Most of the commercial orthoses are only partially realized with AM technologies and then assembled to constitute the final device, with a consequent increase in production phases and costs depending on the particular orthosis to be made [12]. In the literature there are different works about 
wrist orthoses [13], foot orthoses [14] and hip orthoses [15], but there is not much information about the customised elbow orthosis. For this reason, this work focuses on the study of an elbow orthosis. Nowadays there are no design methods specifically focused on orthosis design, which consequently may lead to inefficient devices. However, it is understandable that user needs depend on the patients' medical conditions, the human body segment to be treated, the kinematic behaviour of the orthosis, and these are important criteria that should be taken into account during the conceptual design stage of orthosis development $[7,16]$.

The aim of this work is to setup a method for the design of a new customised elbow orthosis completely manufacturable by $\mathrm{AM}$ and the implementation of generative algorithms for parametric and interactive modelling of $3 \mathrm{D}$ patterns on $\mathrm{CAD}$ models.

The work is structured as follows. In the first step, the $3 \mathrm{D}$ acquisition and CAD modelling of the arm of patient have been performed. The second step consists of the CAD modelling of the customised orthosis. During this step, generative algorithms have been developed for the realization of a Voronoi tessellation and for the creation of patterns with different modules in order to better customise the device. In the third step, results obtained by the FEM analyses performed during the last step were used to validate the design. Finally, a prototype of the elbow orthosis has been realized by means of the SLS technology.

\section{The innovative model of elbow orthosis}

Used in rehabilitation, orthoses increase user's ability to function and improve their quality of life, assisting in different actions and restoring mobility [4, 17]. There are some types of elbow orthoses on the market, most of which are prefabricated and few customised. They can be either with variable or fixed angles and are used to keep the patient's elbow fixed. The basic idea of this work is to design of a new customised elbow orthosis manufactured entirely by AM technology, able to help patients during the rehabilitation phase.

Physiotherapy plays a key role in the post-traumatic and post-surgical elbow rehabilitation process. It is mainly based on the rehabilitation exercise and its function is to promote the full recovery of joint function. The first phase of the physiotherapy pathway aims to restore mobility and treat painful symptoms, through a program of passive manipulations involving flexion-extension movements. The passive exercise serves to train the joint (without tiring the muscles), to counteract the formation of adhesions and to stimulate drainage.

As mentioned, during the rehabilitation of the patient, one of the fundamental exercises is the execution of flexion and

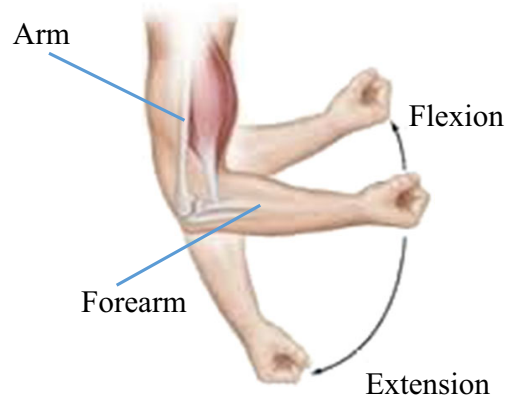

Fig. 1 Extension and flexion movements of the forearm

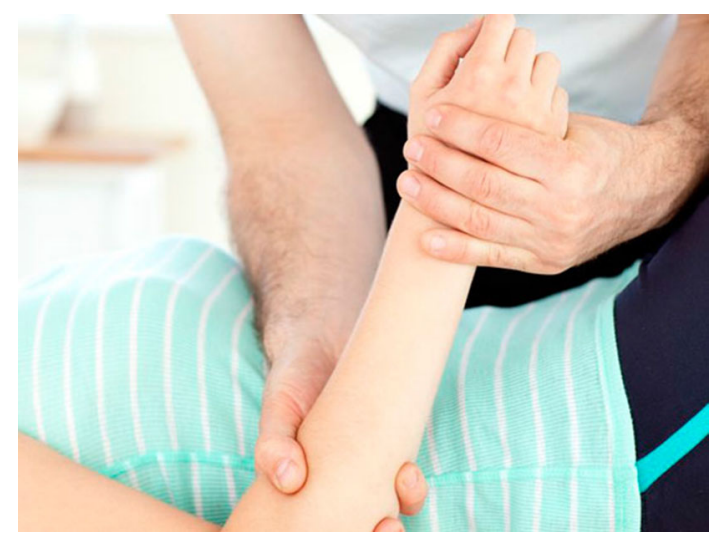

Fig. 2 Rehabilitation exercise with physiotherapist

extension movements of the forearm (Fig. 1) that help to slowly return to the full functionality and mobility of the arm.

Starting from the starting position (angle between arm and forearm of $90^{\circ}$ ) during the flexion and extension movements the physiotherapist opposes resistance. After, to return to the starting position, the patient is eased by the physiotherapist (Fig. 2).

To help the patient during the rehabilitation phase, simulating this exercise, with no support of physiotherapists, it was decided to apply two torsion springs to the orthosis that oppose resistance during the flexion and extension movements and help the patient to move the arm until to reach the starting position.

\section{CAD modelling of elbow orthosis}

To realize a very customised and ergonomic device, the real shape of the patient's arm has been reconstructed through a classical reverse engineering based approach. The first step consists of the $3 \mathrm{D}$ acquisition process and the subsequent CAD modelling of the patient's arm. In this way, a very accurate $\mathrm{CAD}$ model of the arm was obtained (Fig. 3). 


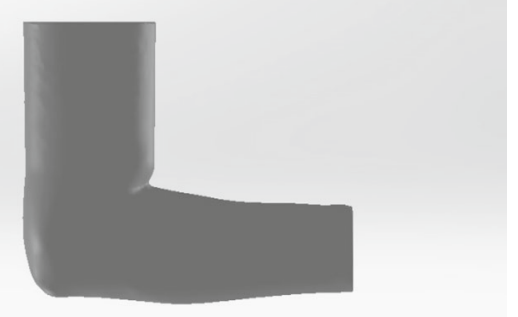

Fig. 3 CAD model of the arm surface

\subsection{CAD modelling of structural part of the orthosis}

Customised orthoses, besides to adapt better to the patient, also allow optimizing their stiffness and flexibility to inhibit some movements and to integrate new morphological characteristics. After the CAD models of the arm were created, the structural parts of the orthosis were modelled.

In order to meet the ergonomics and wearability requirements, the frame of the structural parts of the orthosis were created by applying a $2 \mathrm{~mm}$ offset to the reconstructed surfaces of the arm. In this way, a small clearance between the arm and the orthosis was ensured. Moreover, two longitudinal openings were made along the internal side of the orthosis to increase its fitness and to ensure the possibility for the patient to wear and remove the orthosis easily and independently, with no discomfort at the elbow.

To ensure a suitable stiffness of the orthosis, but at the same time don't add weight to the entire device, preliminary, a thickness of $4 \mathrm{~mm}$ of the structure has been chosen. This value of thickness is usually adopted in commercial orthoses.

Regarding the relative rotation between the arm and the forearm during the extension and flexion movements, initially, it has tried to use two different materials. The idea behind this choice was to entrust the possibility of movement and therefore a greater deformability of the orthosis to the elbow to the softer material, but the results showed many problems. In fact, the realization of a first prototype, by PolyJet technology, using two different materials has shown some defects such as broken part of the structure made with softer material and not perfect bonding between the two materials in some points of the elbow joint (Fig. 4).

For this reason, it was decided to overcome these drawbacks by creating a model of orthosis with a hinge mechanism (Fig. 5).

After the structural parts of the orthosis were modelled, they were assembled into the final CAD model of the orthosis using the SolidWorks software (Fig. 6).

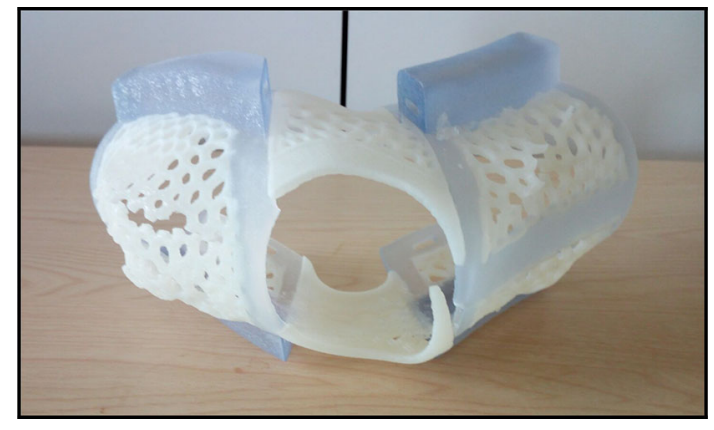

Fig. 4 Prototype realized by PolyJet technology

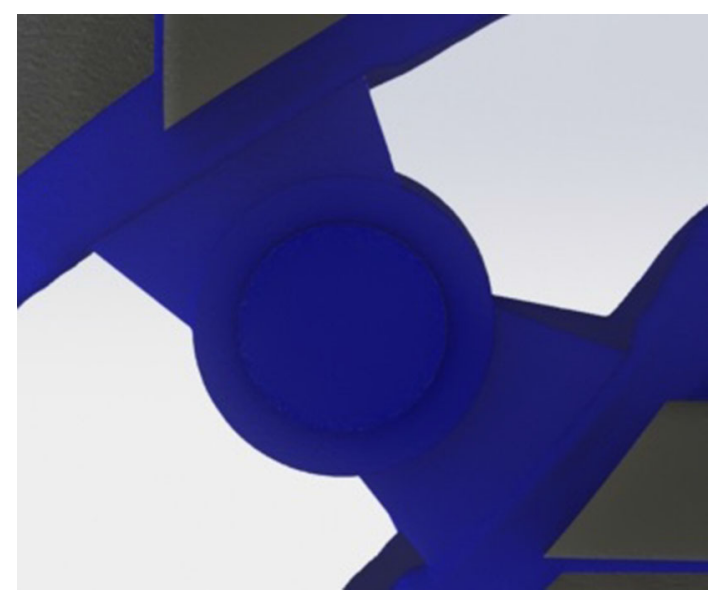

Fig. 5 Hinged mechanism of the elbow orthosis

\subsection{Interactive CAD modelling by generative algorithms}

In the last years, Generative Design (GD) has spread to many fields, such as architecture, jewellery and industrial design. Parametric modelling by GD allows the automated parameter-based generation of any project elements. This means that the generation and modification of the elements within a project is controlled with specific algorithm generated rule sets. Elements are automatically drawn based on user defined algorithms and by changing parameters within the algorithm, a design can be easily controlled in an interactive way $[18,19]$. This approach allows the user to obtain complex design tasks by a semi-automatic modelling process and to customize the resulting geometrical models by interactively modifying certain parameters. Moreover GD, by means of generative algorithms, allows finding solutions to problems that can be encountered with the classic CAD systems when complex shapes, especially when these would be realized using AM techniques [20-22], must be modelled. 
Fig. 6 CAD model of the elbow orthosis

Fig. 7 Block diagram of the implemented generative algorithm
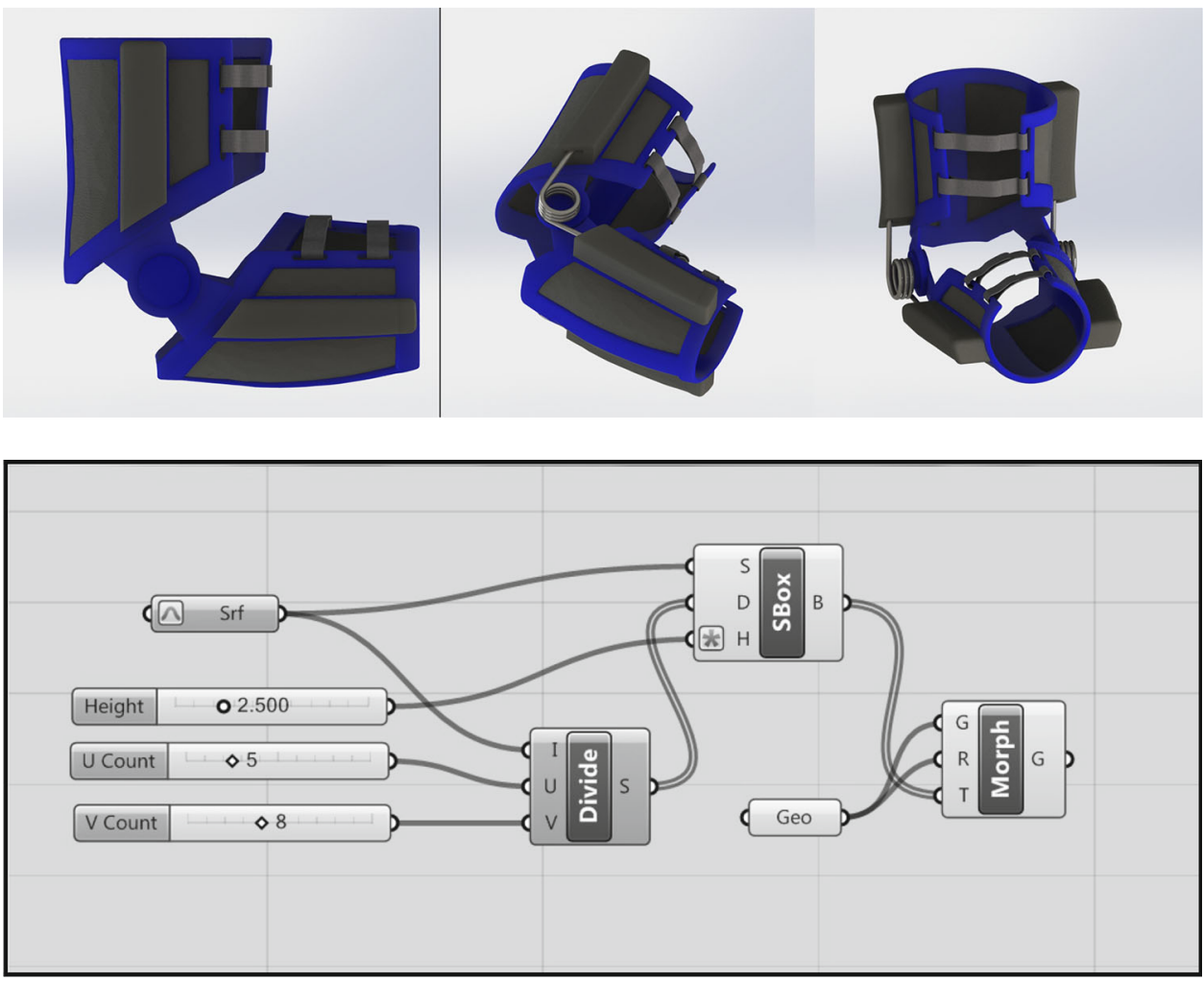

In this work, generative algorithms have been implemented for the modelling of non-structural parts of the orthosis that, in addition to lightening the structure, must make the orthosis pleasant from an aesthetic point of view [23]. Two different algorithms have been developed with Grasshopper [24, 25]; the first allows the creation of patterns on complex surfaces, the second allows realizing a Voronoi tessellation. Both the new proposed approaches allow the modelling and modifying of non-structural parts and, consequently, it is possible to make an interactive evaluation of the created geometries from an aesthetic point of view. It's possible, in fact, to modify the number of elements along the parameterization directions ( $\mathrm{U}$ and $\mathrm{V}$ ), to change the basic geometry and, interactively, to evaluate the obtained designs.

\subsubsection{Patterns}

The developed algorithm allows creating patterns on complex surfaces, so customising the orthosis with different decorative geometries. In particular, this algorithm generates different parallelepipeds on the surface, used as target objects, and maps on these the geometry to repeat. The target parallelepipeds have been generated to morph the component using the "Surface Box" function [24], adapting them to a surface based on the interval indicated by the surface domain and the height of the parallelepiped. The result is the pattern created on the surface that is perfectly adapted to it.
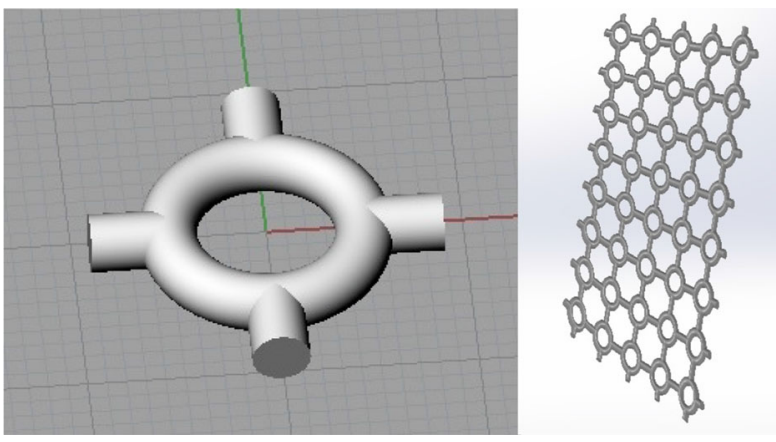

Fig. 8 Example of basic component to repeat (left) and pattern created with generative algorithm

By modifying the basic geometry or the number of elements, the designers can interactively evaluate different solutions in a short time. Figure 7 shows the block diagram of the developed algorithm.

In Fig. 8, as example, basic component to repeat (left) and pattern created with generative algorithm are shown.

In Fig. 9 some examples of patterns created with this algorithm and applied to the elbow orthosis are shown.

\subsubsection{Voronoi tessellation}

Often with some commercial software it is not possible to manage independently the input parameters for the creation of Voronoi tessellations (such as number of points, width of 
Fig. 9 Examples of patterns applied to the orthosis
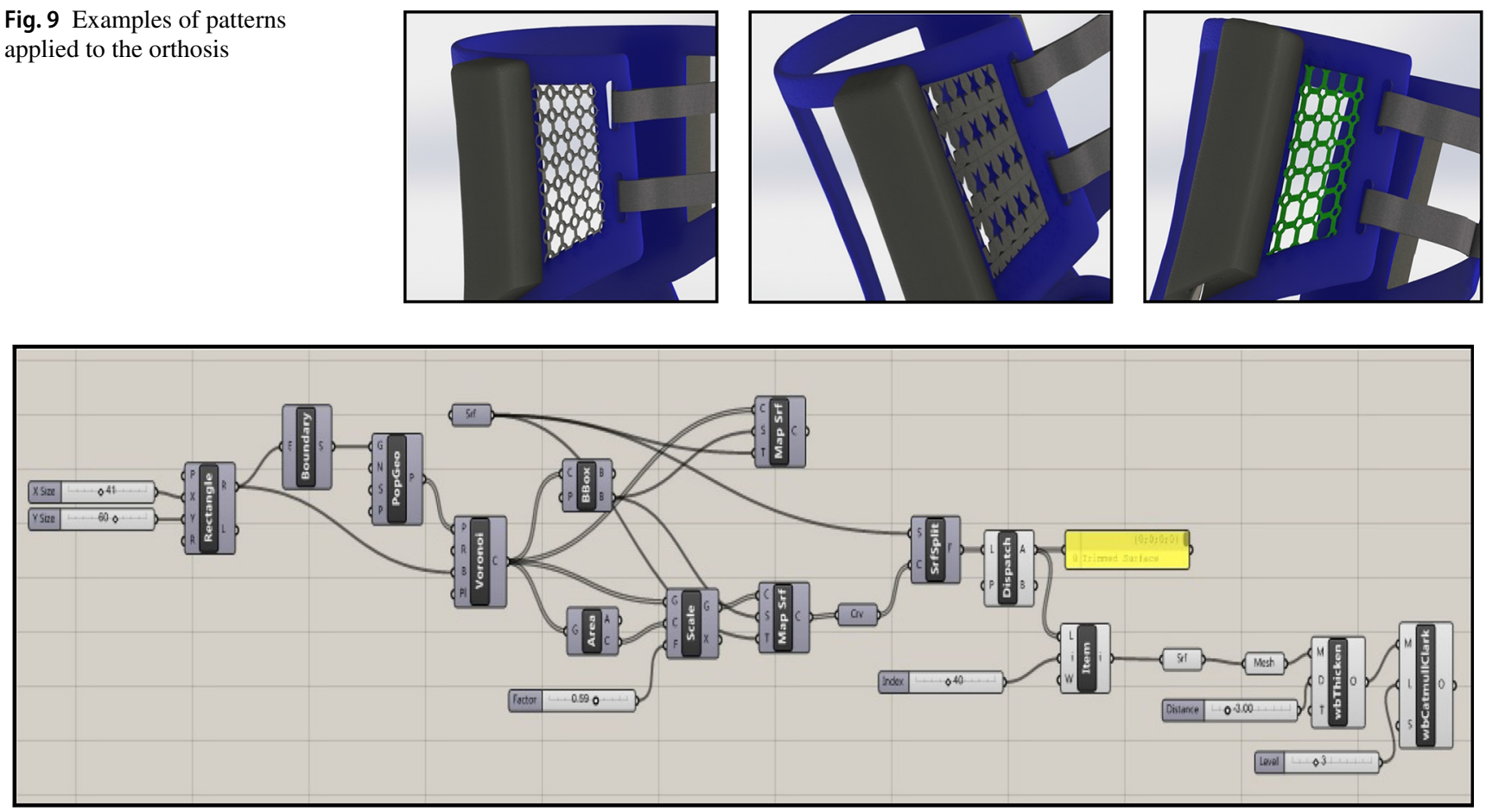

Fig. 10 Block diagram of the implemented generative algorithm for Voronoi tessellation

Fig. 11 CAD model of the elbow orthosis with Voronoi tessellation
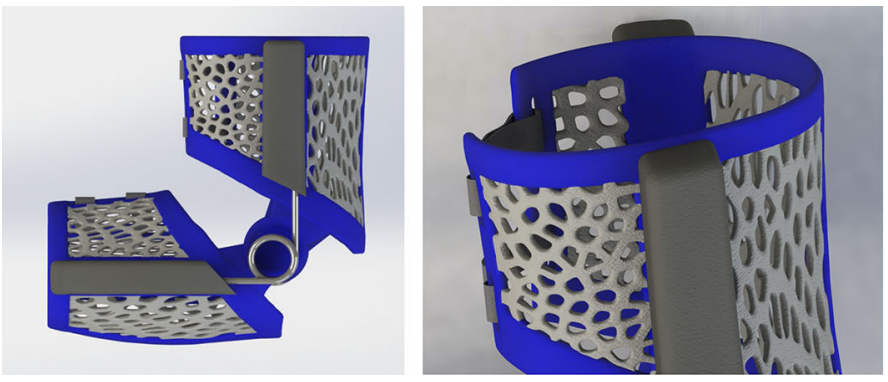

each polygon of the tessellation, etc.). To solve this problem, a generative algorithm has been developed allowing to manage these parameters and to model, parametrically, a Voronoi structure in the custom orthosis. The Voronoi tessellation is initially created on a surface in a plane and is subsequently mapped onto the surface of the orthosis. After, with specific functions, the mesh is generated and exported.

In particular, the creation of the Voronoi tessellation starts from a set of points that represent the centre of the polygons of the tessellation; after, through the Voronoi function, a twodimensional Voronoi tessellation has been created according to the set of points. The structure is mapped onto the surface of the orthosis through the "Map to Surface" function [24], adapting itself to the morphology of the surface. Thanks to a specific function, it is possible to interactively modify the set of points so to obtain different designs. The last step of the algorithm is the creation of mesh and the smoothing of the structure so to eliminate the sharp edges. Figure 10 shows the block diagram of the generative algorithm developed in Grasshopper.

In Fig. 11 the complete CAD model of the orthosis with the Voronoi tessellation is shown.

\section{FEM analyses}

To verify the structural strength of the prototype [26, 27], biomechanical FEM analyses have been executed [3, 28, 29]. These FEM analyses were carried out only on the structural parts of the orthosis. Explicit dynamic simulations were performed to overcome any non-convergence problems due to large displacements during the flexion and extension movements. The simulation time was set to $0.1 \mathrm{~s}$, the initial time step was $0.001 \mathrm{~s}$, an automatic time step approach was used (min time step: $0.0005 \mathrm{~s}$, max time step: $0.005 \mathrm{~s}$ ) and a check on the maximum energy error was also imposed [30]. The 
Table 1 Mechanical properties of PA 2200

\begin{tabular}{llll}
\hline & $\begin{array}{l}\text { Tensile strength } \\
(\mathrm{MPa})\end{array}$ & $\begin{array}{l}\text { Elongation at } \\
\text { break }(\%)\end{array}$ & $\begin{array}{l}\text { Tensile modulus } \\
(\mathrm{MPa})\end{array}$ \\
\hline PA 2200 & 48 & 24 & 1700 \\
\hline
\end{tabular}

arm has been considered as a rigid body. The area near the elbow has been not considered to simplify the FEM model, always ensuring the reliability of the results.

The simulations have been performed to verify the stress state of the orthosis during flexion and extension, applying a test load of $150 \mathrm{~N}$. Although the maximum load in normal conditions of use of an elbow orthosis should be less than $50 \mathrm{~N}$, a much higher test load $(150 \mathrm{~N})$ was chosen to test the model in very hard-working conditions.

10-node tetrahedral elements have been used to mesh the full-scale model of the orthosis. To ensure a very high accuracy of the numerical results and to reduce the gap between numerical simulation and reality, a check on the aspect ratio has been imposed during meshing to maintain all elements within acceptable distortion limits and, moreover, a convergence analysis (convergence threshold value: $5 \%$ ) has been performed to set the most suitable element size. The final mesh consisted of about 2.331 .000 elements. A frictional contact has been imposed at the orthosis and arm interface (friction coefficient: 0.30 [31]), a frictionless contact, instead, has been imposed at the torsion springs and the supports interfaces. Finally, a fixed constraint has been applied to the faces of the arm (in the upper part) which prevents them from moving or deforming. The strips have been modelled as spring elements. FEM analyses were performed using a Dell Precision T7810 workstation with a 24-core Intel Xeon E5-2670 processor and 128 gigabytes of random-access memory.

In Table 1 are reported the mechanical properties of PA 2200 that was considered as elastic linear hardening material.

\subsection{Results}

\subsubsection{Extension movement}

Figure 12 shows Von Mises stress maps during extension.

It can be observed that all the maximum values of the strains are lower than the corresponding limit values. In particular, the maximum value of the Von Mises stress is 22.27 MPa, less than half of the tensile strength of the material (48 MPa).

\subsubsection{Flexion movement}

Figure 13 shows Von Mises stress maps during flexion.

Also in this case, it can be observed that all the maximum values of the deformation and strains are lower than the corresponding limit values. In particular, a maximum elongation value of $2.1 \%$ and a maximum value of the Von Mises equivalent stress of $41 \mathrm{MPa}$ have been obtained.

\section{Prototype realization by additive manufacturing}

For the realization of the orthosis, different materials and different technologies have been investigated in order to identify optimal solutions, considering various criteria. Finally, it was decided to realize the elbow orthosis in PA 2200 by a SLS (Selective Laser Sintering) 3D printer. As case study, only the model of the orthosis with the Voronoi tessellation has been produced. The overall dimensions of the components of the orthosis are approximately $150 \times 120 \times 110 \mathrm{~mm}$ and $185 \times 100 \times 90 \mathrm{~mm}$ respectively for the humeral and ulnar components.

PA 2200 is a fine white powder based on polyamide, used for the manufacture of fully functional pieces with high
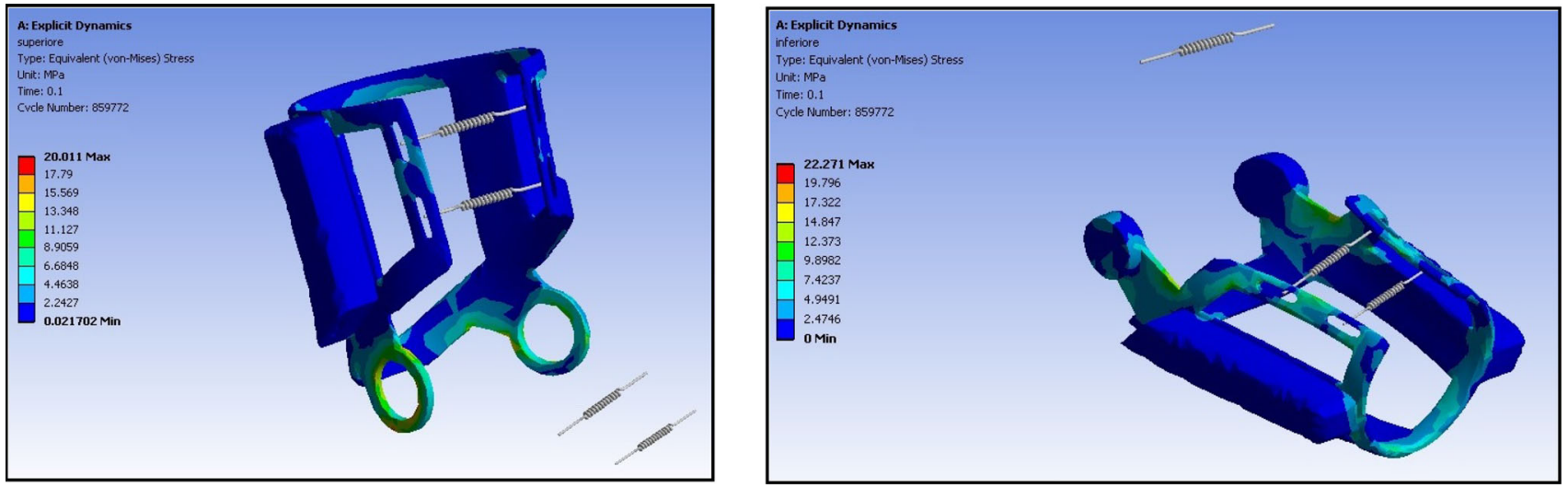

Fig. 12 Maps: Von Mises stress 

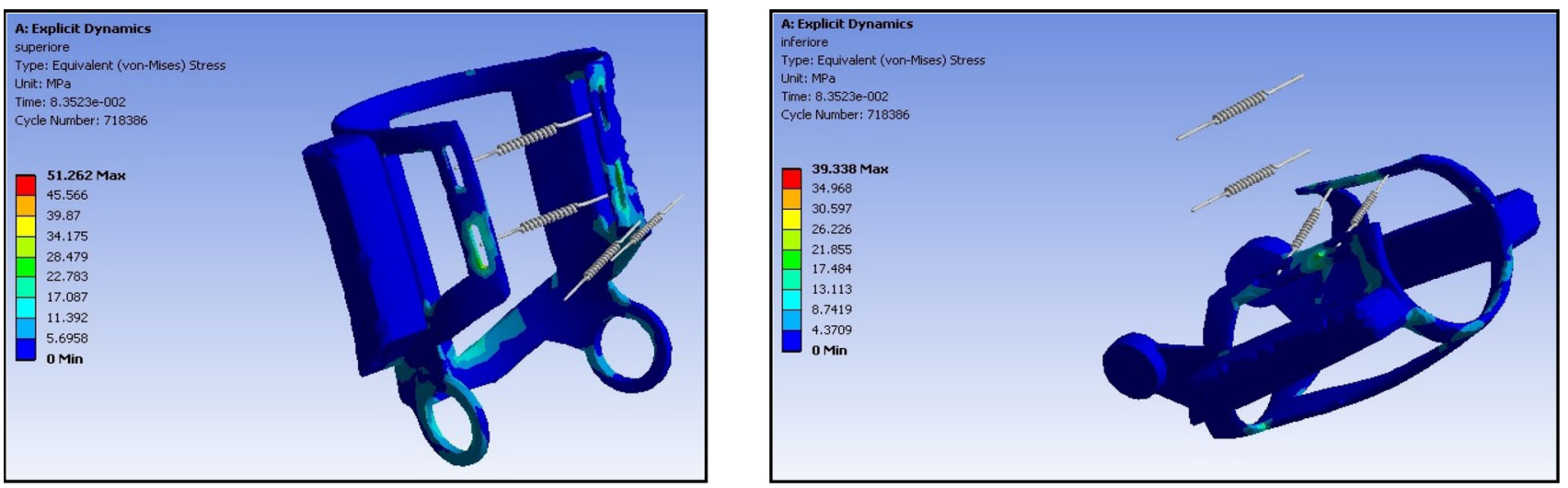

Fig. 13 Maps: Von Mises stress

Fig. 14 Final prototype of the designed elbow orthosis
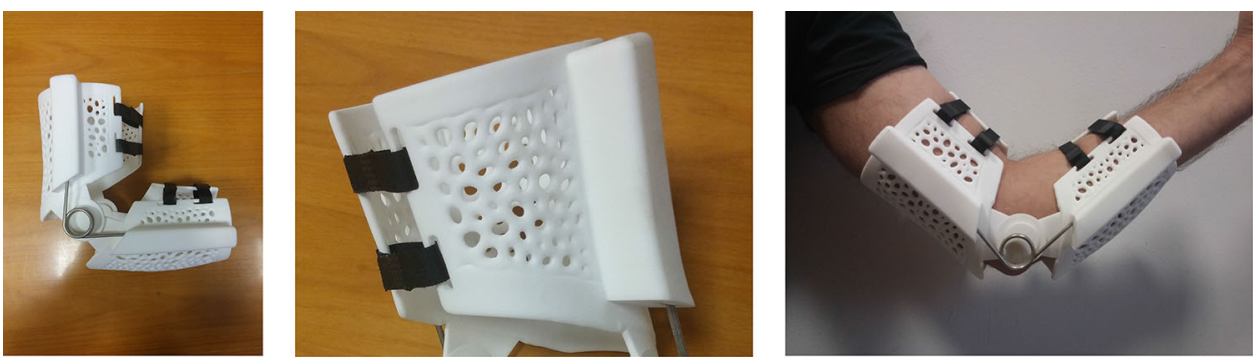

surface quality and exposed to high thermal or mechanical stresses [32, 33].

The EOS FORMIGA P100 printer has been used, which allows the production of small series and customised products with fully functional complex geometry, and ensures a gap between CAD model and real prototype lower than $0.1 \mathrm{~mm}$.

Figure 14 shows the final prototype from which is possible to see the good surface finish obtained with this AM technology.

\section{Conclusions}

In this work a new method for the design of customised orthopaedic devices completely manufacturable by AM is presented. The new developed approach has been effectively tested by designing a customised elbow orthosis. A classical reverse engineering approach has been used to digitalize the patient's arm and the subsequent CAD modelling of the structure of the custom elbow orthosis has been performed. To enhance the aesthetic appeal, two algorithms have been implemented for the creation of 3D patterns and Voronoi tessellations for not structural parts. FEM analyses have been also carried out considering the movements of flexion and extension of the arm and obtained results were used to validate the design. The CAD model of the orthosis created with the new tool has been subsequently used to produce a proto- type of the elbow orthosis by means of the SLS technology. A fully functional prototype having a good surface finish has been obtained.

Finally, it can be stated the developed generative algorithms have been tested and proved to be robust, efficient and able to overcome some drawbacks of commercial software. The new algorithms could meet the expectations of designers and simplify the process of designing of biomedical devices. The new modelling approaches, in fact, allow to interactively model and evaluate different designs in a very simple and fast way. The developed procedure, moreover, could be effectively used for the designing of other kinds of customised devices for orthopaedic applications in an interactive way.

Funding Open access funding provided by Università degli Studi di Palermo within the CRUI-CARE Agreement.

Open Access This article is licensed under a Creative Commons Attribution 4.0 International License, which permits use, sharing, adaptation, distribution and reproduction in any medium or format, as long as you give appropriate credit to the original author(s) and the source, provide a link to the Creative Commons licence, and indicate if changes were made. The images or other third party material in this article are included in the article's Creative Commons licence, unless indicated otherwise in a credit line to the material. If material is not included in the article's Creative Commons licence and your intended use is not permitted by statutory regulation or exceeds the permitted use, you will need to obtain permission directly from the copyright holder. To view a copy of this licence, visit http://creativecomm ons.org/licenses/by/4.0/. 


\section{References}

1. Chen, R.K., Jin, Y., Wensman, J., Shih, A.: Additive manufacturing of custom orthoses and prostheses-a review. Addit. Manuf. 12, 77-89 (2016)

2. Ingrassia, T., Nigrelli, V., Ricotta, V., Nalbone, L., D’Arienzo, A., D'Arienzo, M., Porcellini, G.: A new method to evaluate the influence of the glenosphere positioning on stability and range of motion of a reverse shoulder prosthesis. Inj. Int. J. Care Inj. 50, S12-S17 (2019)

3. Ingrassia, T., Nalbone, L., Nigrelli, V., Ricotta, V., Pisciotta, D.: Biomechanical analysis of the humeral tray positioning in reverse shoulder arthroplasty design. Int. J. Interact. Des. Manuf. 12(2), 651-661 (2018). https://doi.org/10.1007/s12008-017-0418-8

4. Duarte, R., Mesnard, M., Nadeau, J.P.: An innovative design approach to develop external articular medical devices. Int. J. Interact. Des. Manuf. 11, 375-383 (2017). https://doi.org/10.1007/s12 008-016-0341-4

5. Javaid, M., Haleem, A.: Additive manufacturing applications in medical cases: a review literature based. Alex. J. Med. 54(4), 411-422 (2018). https://doi.org/10.1016/j.ajme.2017.09.003

6. Singh, S., Ramakrishna, S.: Biomedical applications of additive manufacturing: present and future. Curr. Opin. Biomed. Eng. 2, $105-115$ (2017)

7. Ricotta, V., Campbell, R., Ingrassia, T., Nigrelli, V.: Additively manufactured textiles and parametric modelling by generative algorithms in orthopaedic applications. Rapid Prototyp. J. 26(5), 827-834 (2020). https://doi.org/10.1108/RPJ-05-2019-0140

8. Bikas, H., Stavropoulos, P., Chryssolouris, G.: Additive manufacturing methods and modelling approaches: a critical review. Int. J. Adv. Manuf. Technol. (2015). https://doi.org/10.1007/s00170-0 15-7576-2

9. Huang, S.H., Liu, P., Mokasdar, A., Hou, L.: Additive manufacturing and its societal impact: a literature review. Int. J. Adv. Manuf. Technol. 67, 1191-1203 (2013). https://doi.org/10.1007/s00170-0 12-4558-5

10. Gao, W., Zhang, Y., Ramanujan, D., Ramania, K., Chen, Y., Williams, C.B., Wang, C.C.L., Shin, Y.C., Zhang, S., Zavattieri, P.D.: The status, challenges, and future of additive manufacturing in engineering. Compute.-Aided Des. 69, 65-89 (2015)

11. Ingrassia, T., Nigrelli, V., Ricotta, V., Tartamella, C.: Process parameters influence in additive manufacturing. In: Eynard, B., Nigrelli, V., Oliveri, S., Peris-Fajarnes, G., Rizzuti, S. (eds.) Advances on Mechanics, Design Engineering and Manufacturing. Lecture Notes in Mechanical Engineering, pp. 261-270. Springer, Cham (2017). https://doi.org/10.1007/978-3-319-45781-9_27

12. Eyers, D.R., Potter, A.T.: Industrial additive manufacturing: a manufacturing systems perspective. Comput. Ind. 92, 208-218 (2017)

13. Paterson, A.M., Donnison, E., Bibb, R.J., Campbell, R.I.: Computer-aided design to support fabrication of wrist splints using 3D printing: a feasibility study. Hand Ther. 19(4), 102-113 (2014). https://doi.org/10.1177/1758998314544802

14. Fantini, M., De Crescenzio, F., Brognara, L., Baldini, N.: Design and rapid manufacturing of a customized foot orthosis: a first methodological study. In: Eynard, B., Nigrelli, V., Oliveri, S., PerisFajarnes, G., Rizzuti, S. (eds.) Advances on Mechanics, Design Engineering and Manufacturing. Lecture Notes in Mechanical Engineering. Springer, Cham (2017). https://doi.org/10.1007/9783-319-45781-9_46

15. Munhoz, R., Da Costa Moraes, C.A., Tanaka, H., Kunkel, M.E.: A digital approach for design and fabrication by rapid prototyping of orthosis for developmental dysplasia of the hip. Res. Biomed. Eng. 32(1), 63-73 (2016). https://doi.org/10.1590/2446-4740.00316

16. Regufe, L., Duarte, R., Ramos, A., Nadeau, J.P., Perry, N., Mesnard, M.: An exhaustive method for researching articular orthosis mechanisms at the conceptual design stage. Procedia CIRP 60, 482-487 (2017)

17. Ingrassia, T., Nalbone, L., Nigrelli, V., Pisciotta, D., Ricotta, V.: Influence of the metaphysis positioning in a new reverse shoulder prosthesis. In: Eynard, B., Nigrelli, V., Oliveri, S., Peris-Fajarnes, G., Rizzuti, S. (eds.) Advances on Mechanics, Design Engineering and Manufacturing. Lecture Notes in Mechanical Engineering, pp. 469-478. Springer, Cham (2017). https://doi.org/10.1007/9783-319-45781-9_47

18. Francalanza, E., Fenech, A., Paul Cutajar, P.: Generative design in the development of a robotic manipulator. Procedia CIRP 67, 244-249 (2018)

19. Eltaweel, A., Su, Y.: Parametric design and daylighting: a literature review. Renew. Sustain. Energy Rev. 73, 1086-1103 (2017)

20. Klahn, C., Leutenecker, B., Meboldt, M.: Design strategies for the process of additive manufacturing. Procedia CIRP 36, 230-235 (2015)

21. Hällgren, S., Pejryd, L., Ekengren, J.: (Re)Design for additive manufacturing. Procedia CIRP 50, 246-251 (2016)

22. Thompson, M.K., Moroni, G., Vaneker, T., Fadel, G., Campbell, R.I., Gibson, I., Bernard, A., Schulz, J., Graf, P., Ahuja, B., Martina, F.: Design for additive manufacturing: trends, opportunities, considerations, and constraints. CIRP Ann. Manuf. Technol. 65(2), 737-760 (2016)

23. Bai, J., Luo, H., Qin, F.: Design pattern modeling and extraction for CAD models. Adv. Eng. Softw. 93, 30-43 (2016)

24. Khabazi, Z.: Generative Algorithms using Grasshopper. (2010)

25. Krish, S.: A practical generative design method. J. Comput.-Aided Des. 43(1), 88-100 (2011)

26. Ingrassia, T., Lombardo, B., Nigrelli, V., Ricotta, V., Nalbone, L., D'Arienzo, A., D'Arienzo, M., Porcellini, G.: Influence of sutures configuration on the strength of tendon-patch joints for rotator cuff tears treatment. Inj. Int. J. Care Inj. 50, S18-S23 (2019)

27. Tumino, D., Ingrassia, T., Nigrelli, V., Pitarresi, G., Urso Miano, V.: Mechanical behavior of a sandwich with corrugated GRP core: numerical modeling and experimental validation. Frat. Integr. Strut. 30, 317-326 (2014)

28. Mirulla, A.I., Bragonzoni, L., Zaffagnini, S., Bontempi, M., Nigrelli, V., Ingrassia, T.: Virtual simulation of an osseointegrated trans-humeral prosthesis: a falling scenario. Inj. Int. J. Care Inj. 49(4), 784-791 (2018)

29. Ingrassia, T., Mancuso, A.: Virtual prototyping of a new intramedullary nail for tibial fractures. Int. J. Interact. Des. Manuf. 7(3), 159-169 (2013)

30. Baron Saiz, C., Ingrassia, T., Nigrelli, V., Ricotta, V.: Thermal stress analysis of different full and ventilated disc brakes. Frat. Integr. Strut. 34, 608-621 (2015). https://doi.org/10.3221/IGF-ESIS.34.6 7

31. Entezari, A., Fang, J., Sue, A., Zhang, Z., Swain, M.V., Li, Q.: Yielding behaviors of polymeric scaffolds with implications to tissue engineering. Mater. Lett. 184, 108-111 (2016)

32. Singh, S., Ramakrishna, S., Singh, R.: Material issues in additive manufacturing: a review. J. Manuf. Process. 25, 185-200 (2017)

33. Srinivas, M., Sridhar Babu, B.: A critical review on recent research methodologies in additive manufacturing. Mater. Today Proc. 4, 9049-9059 (2017)

Publisher's Note Springer Nature remains neutral with regard to jurisdictional claims in published maps and institutional affiliations. 